\title{
A New Distributed Optimization for Community Microgrids Scheduling
}

\author{
Guodong Liu*, Bailu Xiao*, Michael Starke*, Xiaohu Zhang ${ }^{\dagger}$ and Kevin Tomsovic ${ }^{\dagger}$ \\ *Power and Energy Systems Group, Oak Ridge National Laboratory \\ Oak Ridge, Tennessee 37381 \\ Email: liug@ornl.gov,xiaob@ornl.gov,starkemr@ornl.gov \\ ${ }^{\dagger}$ Department of Electrical Engineering and Computer Science \\ University of Tennessee, Knoxville, Tennessee 37996 \\ Email:xzhang46@utk.edu,tomsovic@tennessee.edu
}

\begin{abstract}
This paper proposes a distributed optimization model for community microgrids considering the building thermal dynamics and customer comfort preference. The microgrid central controller (MCC) minimizes the total cost of operating the community microgrid, including fuel cost, purchasing cost, battery degradation cost and voluntary load shedding cost based on the customers' consumption, while the building energy management systems (BEMS) minimize their electricity bills as well as the cost associated with customer discomfort due to room temperature deviation from the set point. The BEMSs and the MCC exchange information on energy consumption and prices. When the optimization converges, the distributed generation scheduling, energy storage charging/discharging and customers' consumption as well as the energy prices are determined. In particular, we integrate the detailed thermal dynamic characteristics of buildings into the proposed model. The heating, ventilation and air-conditioning (HVAC) systems can be scheduled intelligently to reduce the electricity cost while maintaining the indoor temperature in the comfort range set by customers. Numerical simulation results show the effectiveness of proposed model.
\end{abstract}

Keywords-Community microgrids, scheduling, thermal dynamic model, decentralized optimization, alternating direction method of multipliers (ADMM).

\section{INTRODUCTION}

The growth of distributed renewable and/or nonrenewable energy resource installations, emerging utility-scale energy storage, plug-in hybrid electric vehicle use, and demand response are bringing unprecedented opportunities and challenges to the electric distribution system. As these technologies evolve, utilities, end users, manufacturers, and other

This manuscript has been authored by UT-Battelle, LLC under Contract No. DE-AC05-00OR22725 with the U.S. Department of Energy. The United States Government retains and the publisher, by accepting the article for publication, acknowledges that the United States Government retains a nonexclusive, paid-up, irrevocable, world-wide license to publish or reproduce the published form of this manuscript, or allow others to do so, for United States Government purposes. The Department of Energy will provide public access to these results of federally sponsored research in accordance with the DOE Public Access Plan(http://energy.gov/downloads/doe-publicaccess-plan).

This work also made use of Engineering Research Center Shared Facilities supported by the Engineering Research Center Program of the National Science Foundation and the Department of Energy under NSF Award Number EEC-1041877 and the CURENT Industry Partnership Program. participants in distribution system operations are actively transforming the utility operational model. Traditionally, utilities buy energy from the wholesale energy market and sell it to their customers. The whole distribution network is passive and the power flow is unidirectional. However, this paradigm has been changed with the integration of various distributed energy resources and energy storage on the customer side. In fact, utilities are now facing an active distribution network with bidirectional electricity flow. Advanced coordination and integration are necessary for the efficient operation of future distribution system.

One available approach for integrating these technologies is through a microgrid. A microgrid can be defined as a low voltage distribution network comprising various distributed generation (DG), storage devices, and responsive loads that can be operated in both grid-connected and islanded modes [1]. It is connected to the main distribution network at the Point of Common Coupling (PCC), importing or exporting power to the distribution network as well as providing ancillary services, such as, voltage support, to the main distribution grid [2], [3]. A microgrid can also improve local reliability, reduce emissions and contribute to lower cost of energy supply by taking advantage of DG, storage devices and responsive loads [4]. Due to such benefits, the microgrid has attracted growing attention from both academia and industry [5].

A microgrid central controller (MCC) usually performs the scheduling of the microgrid in both grid-connected and islanded modes [5]. The MCC determines the optimal dispatch of DG and exchanged power between microgrid and distribution utility through PCC that minimizes total operating cost subjected to various technical, environmental, reliability and operating constraints. Considerable efforts have been devoted to optimal scheduling and management of microgrids [7]. Scheduling methods for a microgrid in islanded modes are proposed in [8]-[11]. In addition, numerous research and published works are devoted to the scheduling of microgrid in grid-connected mode [12]-[17]. In particular, deterministic models are used in [12] and [13], while stochastic programming models have been developed 
in [14]-[16]. A hybrid stochastic/robust programming model for microgrid scheduling is proposed in [17]. Recently, optimal control schemes for HVAC considering thermal dynamic model of buildings has been proposed in [19]-[20]. Given indoor temperature settings (desirable temperature and allowable temperature deviation) from customers, the HVAC system can precool (or preheat) the building by turning on during low price hours and turning off during high/peak price hours while still maintaining the indoor temperature in allowable range. By integrating the thermal dynamic model of buildings into the microgrid scheduling process and allowing the MCC surrogate control of the HVAC systems, significant savings in electricity cost can be achieved by coordinating the DG and battery operation, HVAC systems consumption and energy purchasing/selling at PCC while preserving customers' comfort. Nevertheless, the HVAC system was modeled as a continuous controllable load, while in practical cases, most HVAC systems can only be switched on and off.

In these literatures, customers' consumption is mostly considered as constant values or responsive loads, which are directly controlled by the MCC. In fact, the consumers and house/building owners are generally very reluctant to allow MCC directly control their appliances (e.g., HVAC systems) due to various issues, such as psychological safety, privacy protection, etc. For this reason, distributed optimization has been proposed to schedule residential loads in [21][23]. Dual decomposition algorithm is used to decouple complicating variables in the power balance constraint, so the MCC as well as each house can schedule their resources and consumption separately. At residential level, the BEMS in each house schedules its consumption based on price signals distributed by MCC. Then, the MCC schedules the distributed generation and energy storage based on the customers' consumption and update the price signals. This process repeats until the power balance constraint is satisfied, i.e., total generation equals total consumption. The MCC adjusts prices (Lagrangian multipliers) in each iteration, rather than allocating the consumption of customers directly. To guarantee the convergence, all dual subproblems are assumed convex and finite in [21]-[23]. However, the dual subproblems are actually nonconvex due to the integer constraints (on/off of generators and HVAC systems), which cause non-convergence of the algorithm [24].

For this reason, a new distributed optimization model for community microgrids operation considering the building thermal dynamics and customer comfort preference is proposed in this paper. Dual decomposition algorithm is used to decouple the community microgrid scheduling problem into parallel subproblems of MCC and BEMSs. Considering the nonconvexity of dual subproblems, the alternating direction method of multipliers (ADMM) is introduced to guarantee the convergence [24]. Also, detailed thermal dynamic characteristics of houses and HVAC system models are integrated into the BEMS scheduling model. The main contributions of this paper are as follows:

- Integrating the building thermal dynamics and HVAC systems controller into BEMSs and reducing operating cost of microgrids;

- Using ADMM to guarantee the convergence of the distributed optimization with nonconvex dual subproblems; and

- Validating the proposed distributed optimization with numerical simulation results.

The rest of this paper is organized as follows. In Section II, the model of community microgrid and thermal dynamic model of buildings are presented. Based on that, the centralized community microgrid scheduling model considering the building thermal dynamics and customer comfort preference is formulated in Section III. Then, the distributed optimization model is proposed in Section IV. In Section V, results of case study on a community microgrid are presented. Finally, conclusions are given in Section VI.

\section{System Modeling}

\section{A. Community Microgrid Model}

In this paper, we consider a community microgrid that consists of various distributed generation (PV panels and wind turbines, microturbines, fuel cells, diesel generations, etc.), energy storage (e.g., batteries) and a number of houses. In each house, BEMS schedules the appliances to minimize the utility bill while mitigating the impact on user's comfort. The loads can be categorized based on whether they can be scheduled to earlier/later times. They are labeled responsive and non-responsive loads. Typical non-responsive loads includes refrigerator-freezer, electric stove, lighting, TV, computer, etc. Rescheduling these loads will cause significant inconvenience for the customers. Thus, they are taken as non-sensitive to the energy price and non-schedulable. On the contrary, dishwasher, cloth washer/dryer, HVAC, water heater and electric vehicle (EV) have prescribed energy requirements, which are flexible over certain time slots. Therefore, based on the electricity price and the residents' comfort, the BEMS decides when to turn the device on and off. So, they are taken as sensitive to energy price and schedulable. Particularly, HVAC is indeed thermal load with temperature profile, which must be kept within minimum and maximum temperature limits. Due to the thermal inertia of a house, the indoor temperature changes quite slow. This gives the microgrid controller or BEMS extra flexibility to schedule the HVAC. Modeling HVAC as a thermal load requires building the thermal dynamic model of each house, which needs to consider many factors, such as effective window area, the fraction of solar irradiation entering the inner walls and floor, the thermal capacitance and resistance parameters of the house. It is much more complicated compared to other loads. For this reason, we divide the load 


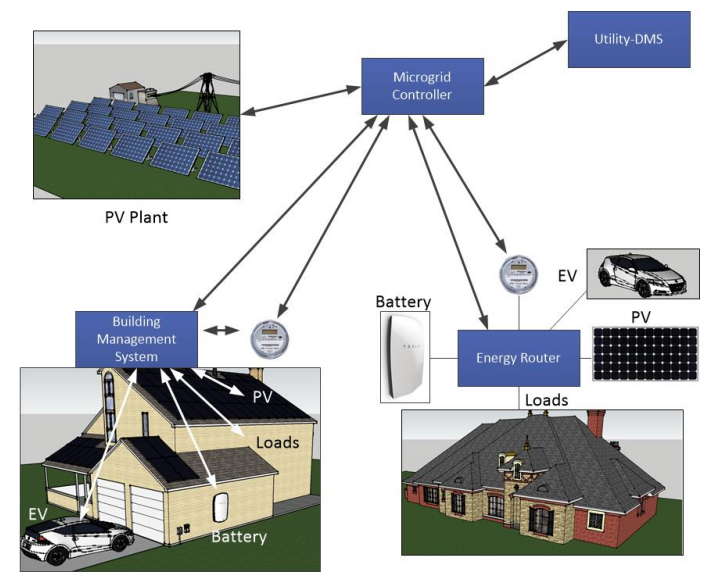

Figure 1: Example community microgrid

into HVAC loads and non-HVAC loads. In addition, rooftop PV panels, small batteries and EVs may also exist.

A building energy management system (BEMS) is equipped for each house to communicate with the microgrid controller. Generally, the objective of the microgrid controller is to minimize the total cost of operating the community microgrid by coordinating the DG and battery operation, customers' consumption and energy purchasing/selling at PCC while preserving customers' comfort. In particular, we assume the microgrid controller collects customer indoor temperature settings (desirable temperature and allowable temperature deviation) and other required information from each BEMS. Based on customer requirements, electricity demand, renewable generation and price information at PCC, microgrid controller decides the power output of controllable DG, charging/discharging power of batteries, purchasing/selling power at PCC and consumption of HVAC systems as well as other controllable loads over a scheduling period (e.g., 24 hours). An example of the community microgrid under consideration is shown in Fig. 1.

\section{B. HVAC System Model}

HVAC systems are typically controlled by thermostats to maintain the house indoor temperature in a comfortable range. Depending on customers' preference, the desirable indoor temperature and allowable temperature range will be preset. Inside the thermostats, a temperature controlled relay circuit is used to switch the HVAC on and off according to the temperature detected by the temperature sensor. The output from the thermostats is either on or off. For cooling control, the HVAC system is switched on when the indoor temperature reaches the ceiling of the allowed temperature range. It will be switched off when the indoor temperature falls below the floor of the allowed temperature range. This autonomous device has been widely used in buildings and houses where automatic temperature control is needed.

Different from the autonomous temperature control above, we assume the desirable temperature and allowable temperature range set by customers are forwarded to the community microgrid controller by the BEMS and the microgrid controller takes surrogate control of HVAC systems. Since indoor temperature changes quite slowly due to the house thermal inertia, a house can be considered as a thermal storage facility. This gives the microgrid controller certain flexibility to schedule the consumption of HVAC system. Specially, a microgrid controller can switch on HVAC systems during low price or high renewable generation intervals to precool (or preheat) the house and switch off them in opposite cases while still maintaining the indoor temperature in allowable range. As a result, it is expected that significant savings in electricity cost can be achieved compared to autonomous temperature control [19]-[20].

\section{Building Thermal Dynamic Model}

Considering the impact of ambient temperature, solar irradiance and HVAC systems, a third order state-space model is employed to describe the thermal dynamic characteristics of a house [25] as:

$$
\boldsymbol{T}_{h, t+1}=\boldsymbol{A}_{h} \boldsymbol{T}_{h, t}+\boldsymbol{B}_{h} \boldsymbol{U}_{h, t} \quad \forall h, \forall t
$$

where $\boldsymbol{T}_{h, t}=\left[T_{h t}^{\mathrm{In}}, T_{h t}^{M}, T_{h t}^{E}\right]$ is the state vector. $\boldsymbol{U}_{h, t}=$ $\left[T_{t}^{A}, \Phi_{t}, u_{h t} \eta_{h} \sigma_{h} P_{h}^{H}\right]$ is the input vector, where $\sigma_{h}=1$ corresponds to winter heating and $\sigma_{h}=-1$ for summer cooling. The coefficients of matrices $\boldsymbol{A}_{h}$ and $\boldsymbol{B}_{h}$ of a house $h$ can be calculated based on the effective window area, the fraction of solar irradiation entering the inner walls and floor, the thermal capacitance and resistance parameters of the house, and the sampling time, which is the time solution of the optimization horizon. More details on this building thermal dynamic model can be found in [25]. The constraints on house indoor temperature are:

$$
T_{h t}^{D}-\delta_{h t} \leq T_{h t}^{\mathrm{In}} \leq T_{h t}^{D}+\delta_{h t}
$$

\section{Centralized Community Microgrid SCHEDULING}

The community microgrid considered in this paper consists of distributed generators (e.g., diesel generators, microturbines and fuel cells), renewable generation (e.g., wind turbines and PV panels), energy storage (e.g., battery systems) and load (HVAC and non-HVAC). The distributed generators are considered dispatchable units, which can be controlled by a MCC to provide dispatched power. Depending on unit type, dispatchable units are subject to various constraints, such as, capacity limits, ramping rates, minimum power output limits, minimum on/off time, and so on. In contrast, renewable generation, such as, wind turbines and PV panels, are taken as non-dispatchable units, which depend on the meteorological conditions of wind speed, ambient temperature and solar irradiance. Loads are divided to HVAC loads and non-HVAC loads. Each HVAC load is associated 
with a house or building. The community microgrid is also connected to the external utility system via the PCC characterized by a forecasted day-ahead market price known for a time window of 24 hours. Under this assumption, the operation objective of the community microgrid is to minimize a virtual cost associated with the system operating cost and customer discomfort as in (3). Specifically, the first and second line is the fuel cost of DGs (including DG start-up cost); the third line is the energy purchasing/selling cost/benefit from distribution grid; the fourth and fifth lines are cost of battery degradation and load shedding; and the sixth line is the discomfort cost of customers due to the deviations from customer desired temperature.

$$
\begin{aligned}
\min & \sum_{t=1}^{N_{T}} \sum_{i=1}^{N_{G}}\left[\sum_{m=1}^{N_{I}} \lambda_{i t}^{P}(m) p_{i t}(m)+\kappa_{i} u_{i t}\right] \\
& +\sum_{t=1}^{N_{T}} \sum_{i=1}^{N_{G}} S U_{i t}\left(u_{i t}, u_{i, t-1}\right) \\
& +\sum_{t=1}^{N_{T}} \lambda_{t}^{\mathrm{PCC}} P_{t}^{\mathrm{PCC}} \\
& +\sum_{t=1}^{N_{T}} \sum_{b=1}^{N_{B}} C_{b t}\left(P_{b t}^{\mathrm{C}}+P_{b t}^{\mathrm{D}}\right) \\
& +\sum_{t=1}^{N_{T}} V_{t}^{\mathrm{LS}} P_{t}^{\mathrm{LS}} \\
& +\sum_{t=1}^{N_{T}} \sum_{h=1}^{N_{H}} \omega_{h t}\left|T_{h t}^{\mathrm{In}}-T_{h t}^{D}\right|
\end{aligned}
$$

The objective function must be minimized subject to several constraints.

$$
\begin{gathered}
P_{i t}=\sum_{m=1}^{N_{I}} p_{i t}(m)+u_{i t} P_{i}^{\min } \quad \forall i, \forall t \\
0 \leq p_{i t}(m) \leq p_{i t}^{\max }(m) \quad \forall i, \forall t, \forall m \\
P_{i}^{\min } u_{i t} \leq P_{i t} \leq P_{i}^{\max } u_{i t} \quad \forall i, \forall t \\
0 \leq P_{b t}^{\mathrm{C}} \leq P_{b}^{\mathrm{C}, \max } u_{b t}^{\mathrm{C}} \quad \forall b, \forall t \\
0 \leq P_{b t}^{\mathrm{D}} \leq P_{b}^{\mathrm{D}, \max } u_{b t}^{\mathrm{D}} \quad \forall b, \forall t \\
u_{b t}^{\mathrm{C}}+u_{b t}^{\mathrm{D}} \leq 1 \quad \forall b, \forall t \\
S O C_{b t}=C_{b, t-1}+P_{b t}^{\mathrm{C}} \eta_{b}^{\mathrm{C}} \triangle t-P_{b t}^{\mathrm{D}} \frac{1}{\eta_{b}^{\mathrm{D}}} \triangle t \quad \forall b, \forall t \\
S O C_{b t}^{\min } \leq S O C_{b t} \leq S O C_{b t}^{\max } \quad \forall b, \forall t \\
0 \leq P_{t}^{\mathrm{LS}} \leq P_{t}^{\mathrm{LS}, \max } \quad \forall t \\
\sum_{i=1}^{N_{G}} P_{i t}+P_{t}^{W}+P_{t}^{\mathrm{PV}}+P_{t}^{\mathrm{PCC}}+\sum_{b=1}^{N_{B}}\left(P_{b t}^{\mathrm{D}}-P_{b t}^{\mathrm{C}}\right) \\
=\sum_{j=1}^{N_{D}} P_{j t}+\sum_{h=1}^{N_{H}} u_{h t} P_{h}^{H}-P_{t}^{\mathrm{LS}} \quad \forall t
\end{gathered}
$$

For DGs, constraints (4) and (5) approximate the production cost of dispatchable generators by blocks. Constraint (6) forces the output of DG to be zero if it is not committed. Additionally, each unit is subject to its own operating constraints, such as, minimum up and down time, initial condition, ramping rates and so on. Due to the small capacity of generators in the community microgrids, the minimum up and down time as well as the ramping rates are normally neglected. Nevertheless, detailed and standard mathematical formulations of these constraints can be found [26]. For batteries, constraints (7) and (8) are the maximum charging/discharging power of a battery. These two states are mutually exclusive, which is ensured by (9). The battery state of charge (SOC) is defined by (10) and the limit of SOC is enforced by (11). The amount of load curtailment at time $t$ is limited by constraint (12). The energy balance is enforced by (13). The total of the electricity produced by dispatchable generators, wind, PV, batteries and power purchased at PCC must be equal to the summation of HVAC load and non-HVAC load minus the load curtailed in the microgrid. Most importantly, the thermal dynamic equation of houses (1) and house indoor temperature limits (2) should also be considered.

The proposed optimization can be reformulated into mixed-integer linear programming (MILP). All logical terms that appear in the objective function are recast into mixedinteger linear form. For the objective function, the startup cost of DGs (line 2) can be recast into mixed-integer linear form as in [26]. The expression of customer discomfort cost (line 6) can be reformulated into linear format as in (14) - (16) by introducing an auxiliary variable $X_{h t}$, which represents the absolute value of temperature deviation. Thus, the proposed optimization becomes a MILP, which can be solved efficiently by commercial solvers.

$$
\begin{gathered}
X_{h t} \geq T_{h t}^{\mathrm{In}}-T_{h t}^{D} \quad \forall h, \forall t \\
X_{h t} \geq T_{h t}^{D}-T_{h t}^{\mathrm{In}} \quad \forall h, \forall t \\
X_{h t} \geq 0 \quad \forall h, \forall t
\end{gathered}
$$

\section{Distributed COMmunity Microgrid SCHEDULING}

The centralized community microgrid scheduling model presented in Section III seems straight forward and easy to solve. However, this model has two disadvantages. First of all, the house/building appliances (e.g., HVAC systems) have to be directly controlled by the MCC, while customers generally want to preserve their privacy and may wish to withhold detailed information on energy consumption to the utility company. Secondly, the dimension of the centralized optimization increases significantly as the number of customers increase. As a result, the solution efficiency will be compromised. For these reasons, we proposed to breakdown the centralized optimization by dual decomposition and obtain a distributed parallel optimization. 
The centralized optimization model (1)-(16) has a separable structure. Therefore, it can be solved in a distributed way through dual decomposition and sub-gradient method. The Lagrangian function for (3) is given as (17), where $\lambda_{t}$ denotes the Lagrangian multiplier corresponding to constraint (13). The Lagrangian minimization (17), is easily seen to be decomposed to optimizations at the microgrid level and customers.

$$
\begin{aligned}
\min & \sum_{t=1}^{N_{T}} \sum_{i=1}^{N_{G}}\left[\sum_{m=1}^{N_{I}} \lambda_{i t}^{P}(m) p_{i t}(m)+\kappa_{i} u_{i t}\right] \\
& +\sum_{t=1}^{N_{T}} \sum_{i=1}^{N_{G}} S U_{i t}\left(u_{i t}, u_{i, t-1}\right)+\sum_{t=1}^{N_{T}} \lambda_{t}^{\mathrm{PCC}} P_{t}^{\mathrm{PCC}} \\
& +\sum_{t=1}^{N_{T}} \sum_{b=1}^{N_{B}} C_{b t}\left(P_{b t}^{\mathrm{C}}+P_{b t}^{\mathrm{D}}\right)+\sum_{t=1}^{N_{T}} V_{t}^{\mathrm{LS}} P_{t}^{\mathrm{LS}} \\
& +\sum_{t=1}^{N_{T}} \sum_{h=1}^{N_{H}} \omega_{h t}\left|T_{h t}^{\mathrm{In}}-T_{h t}^{D}\right| \\
& -\sum_{t=1}^{N_{T}} \lambda_{t}\left[\sum_{i=1}^{N_{G}} P_{i t}+P_{t}^{W}+P_{t}^{\mathrm{PV}}+P_{t}^{\mathrm{PCC}}+P_{t}^{\mathrm{LS}}\right. \\
& \left.+\sum_{b=1}^{N_{B}}\left(P_{b t}^{\mathrm{D}}-P_{b t}^{\mathrm{C}}\right)-\sum_{j=1}^{N_{D}} P_{j t}-\sum_{h=1}^{N_{H}} u_{h t} P_{h}^{H}\right]
\end{aligned}
$$

$$
\text { s.t. } \quad(1)(2),(4)-(12), \text { and }(14)-(16)
$$

For a convex optimization problem, dual decomposition can converge to a global optimal solution. However, when the problem is not convex, such as (17), even finding a feasible solution becomes difficult. For this reason, the augmented Lagrangian methods were developed in part to bring robustness to the dual ascend method, and in particular, to yield convergence without assumptions like strict convexity or finiteness of (3). The augmented Lagrangian for (3) is given as (18), where $\rho>0$ is called the penalty parameter. Note (17) is the standard Lagrangian for the problem. Problem (18) is clearly equivalent to the original problem (1)-(16), since for any feasible solution, the terms added to the objective are zero.

$$
\begin{aligned}
\min \quad & \sum_{t=1}^{N_{T}} \sum_{i=1}^{N_{G}}\left[\sum_{m=1}^{N_{I}} \lambda_{i t}^{P}(m) p_{i t}(m)+\kappa_{i} u_{i t}\right] \\
& +\sum_{t=1}^{N_{T}} \sum_{i=1}^{N_{G}} S U_{i t}\left(u_{i t}, u_{i, t-1}\right)+\sum_{t=1}^{N_{T}} \lambda_{t}^{\mathrm{PCC}} P_{t}^{\mathrm{PCC}} \\
& +\sum_{t=1}^{N_{T}} \sum_{b=1}^{N_{B}} C_{b t}\left(P_{b t}^{\mathrm{C}}+P_{b t}^{\mathrm{D}}\right)+\sum_{t=1}^{N_{T}} V_{t}^{\mathrm{LS}} P_{t}^{\mathrm{LS}} \\
& +\sum_{t=1}^{N_{T}} \sum_{h=1}^{N_{H}} \omega_{h t}\left|T_{h t}^{\mathrm{In}}-T_{h t}^{D}\right|
\end{aligned}
$$

$$
\begin{aligned}
& -\sum_{t=1}^{N_{T}} \lambda_{t}\left[\sum_{i=1}^{N_{G}} P_{i t}+P_{t}^{W}+P_{t}^{\mathrm{PV}}+P_{t}^{\mathrm{PCC}}+P_{t}^{\mathrm{LS}}\right. \\
& \left.+\sum_{b=1}^{N_{B}}\left(P_{b t}^{\mathrm{D}}-P_{b t}^{\mathrm{C}}\right)-\sum_{j=1}^{N_{D}} P_{j t}-\sum_{h=1}^{N_{H}} u_{h t} P_{h}^{H}\right] \\
& +\frac{\rho}{2} \| \sum_{i=1}^{N_{G}} \mathbf{P}_{i}+\mathbf{P}^{W}+\mathbf{P}^{\mathrm{PV}}+\mathbf{P}^{\mathrm{PCC}}+\mathbf{P}^{\mathrm{LS}} \\
& +\sum_{b=1}^{N_{B}}\left(\mathbf{P}_{b}^{\mathrm{D}}-\mathbf{P}_{b}^{\mathrm{C}}\right)-\sum_{j=1}^{N_{D}} \mathbf{P}_{j}-\sum_{h=1}^{N_{H}} \mathbf{u}_{h}^{(k)} \mathbf{P}_{h}^{H} \|_{2}^{2}
\end{aligned}
$$

s.t.

$$
\text { (1) }(2),(4)-(12) \text {, and (14) - (16) }
$$

The alternating direction method of multipliers (ADMM) can be viewed as an attempt to blend the decomposability of dual decomposition with the superior convergence properties of augmented Lagrangian methods for constrained optimization [24]. It takes the form of a decomposition-coordination procedure, in which the solutions to small local subproblems are coordinated to find a solution to a large global problem. First of all, the augmented Lagrangian problem (18) is decomposed to suboptimizations at the microgrid level as (19) and for each customer as (20). For each iteration, problem (19) and (20) are solved, then the dual variables are updated uses a step size equal to the penalty parameter $\rho$. This procedure will be continued until it converges to a optimum.

$$
\begin{aligned}
\min \quad & \sum_{t=1}^{N_{T}} \sum_{i=1}^{N_{G}}\left[\sum_{m=1}^{N_{I}} \lambda_{i t}^{P}(m) p_{i t}(m)+\kappa_{i} u_{i t}\right] \\
& +\sum_{t=1}^{N_{T}} \sum_{i=1}^{N_{G}} S U_{i t}\left(u_{i t}, u_{i, t-1}\right)+\sum_{t=1}^{N_{T}} \lambda_{t}^{\mathrm{PCC}} P_{t}^{\mathrm{PCC}} \\
& +\sum_{t=1}^{N_{T}} \sum_{b=1}^{N_{B}} C_{b t}\left(P_{b t}^{\mathrm{C}}+P_{b t}^{\mathrm{D}}\right)+\sum_{t=1}^{N_{T}} V_{t}^{\mathrm{LS}} P_{t}^{\mathrm{LS}} \\
& -\sum_{t=1}^{N_{T}} \lambda_{t}^{(k)}\left[\sum_{i=1}^{N_{G}} P_{i t}+P_{t}^{W}+P_{t}^{\mathrm{PV}}+P_{t}^{\mathrm{PCC}}+P_{t}^{\mathrm{LS}}\right. \\
& \left.+\sum_{b=1}^{N_{B}}\left(P_{b t}^{\mathrm{D}}-P_{b t}^{\mathrm{C}}\right)-\sum_{j=1}^{N_{D}} P_{j t}-\sum_{h=1}^{N_{H}} u_{h t}^{(k)} P_{h}^{H}\right] \\
& +\frac{\rho}{2} \| \sum_{i=1}^{N_{G}} \mathbf{P}_{i}+\mathbf{P}^{W}+\mathbf{P}^{\mathrm{PV}}+\mathbf{P}^{\mathrm{PCC}}+\mathbf{P}^{\mathrm{LS}} \\
& +\sum_{b=1}^{N_{B}}\left(\mathbf{P}_{b}^{\mathrm{D}}-\mathbf{P}_{b}^{\mathrm{C}}\right)-\sum_{j=1}^{N_{D}} \mathbf{P}_{j}-\sum_{h=1}^{N_{H}} \mathbf{u}_{h}^{(k)} \mathbf{P}_{h}^{H} \|_{2}^{2}
\end{aligned}
$$




$$
\begin{aligned}
& \min \quad \sum_{t=1}^{N_{T}} \omega_{h t}\left|T_{h t}^{\mathrm{In}}-T_{h t}^{D}\right| \\
& -\sum_{t=1}^{N_{T}} \lambda_{t}^{(k)}\left[\sum_{i=1}^{N_{G}} P_{i t}^{(k)}+P_{t}^{W}+P_{t}^{\mathrm{PV}}+P_{t}^{\mathrm{PCC},(\mathrm{k})}\right. \\
& +P_{t}^{\mathrm{LS},(\mathrm{k})}+\sum_{b=1}^{N_{B}}\left(P_{b t}^{\mathrm{D},(\mathrm{k})}-P_{b t}^{\mathrm{C},(\mathrm{k})}\right) \\
& \left.-\sum_{j=1}^{N_{D}} P_{j t}-\sum_{h=1}^{N_{H}} u_{h t} P_{h}^{H}\right] \\
& +\frac{\rho}{2} \| \sum_{i=1}^{N_{G}} \mathbf{P}_{i}^{(k)}+\mathbf{P}^{W}+\mathbf{P}^{\mathrm{PV}}+\mathbf{P}^{\mathrm{PCC},(k)} \\
& +\mathbf{P}^{\mathrm{LS},(\mathrm{k})}+\sum_{b=1}^{N_{B}}\left(\mathbf{P}_{b}^{\mathrm{D},(\mathrm{k})}-\mathbf{P}_{b}^{\mathrm{C},(\mathrm{k})}\right) \\
& -\sum_{j=1}^{N_{D}} \mathbf{P}_{j}-\sum_{h=1}^{N_{H}} \mathbf{u}_{h} \mathbf{P}_{h}^{H} \|_{2}^{2} \\
& \text { s.t. } \quad(1)(2) \text {, and (14) }-(16) \\
& \lambda_{t}^{(k+1)}=\lambda_{t}^{(k)}+\rho\left[\sum_{i=1}^{N_{G}} P_{i t}^{(k)}+P_{t}^{W}+P_{t}^{\mathrm{PV}}+P_{t}^{\mathrm{PCC},(\mathrm{k})}\right. \\
& +P_{t}^{\mathrm{LS},(k)}+\sum_{b=1}^{N_{B}}\left(P_{b t}^{\mathrm{D},(\mathrm{k})}-P_{b t}^{\mathrm{C},(\mathrm{k})}\right) \\
& \left.-\sum_{j=1}^{N_{D}} P_{j t}-\sum_{h=1}^{N_{H}} u_{h t}^{(k)} P_{h}^{H}\right]
\end{aligned}
$$

For the proposed distributed scheduling model, the MCC broadcasts the updated Lagrange multipliers and the difference between the total generation at microgrid level as well as the total consumption submitted by customers to all residential smart meters through the advanced metering infrastructure (AMI). For each customer, problem (21) is solved and the total consumption is sent back the MCC. In this way, customers don't reveal the individual appliance consumption or battery storage profiles. The diagram of this distributed scheduling method as well as the exchange of information between MCC and BEMSs is shown in Fig. 2 In ADMM, the variables at microgrid level and customer level (BEMS) are updated in an alternating or sequential fashion, which accounts for the term alternating direction. It should be noted that the ADMM ensures the convergence of the distributed optimization, but same as other nonconvex optimization, the global optimum cannot be guaranteed.

\section{Case Studies}

The proposed community microgrid scheduling model is demonstrated on the modified ORNL Distributed Energy

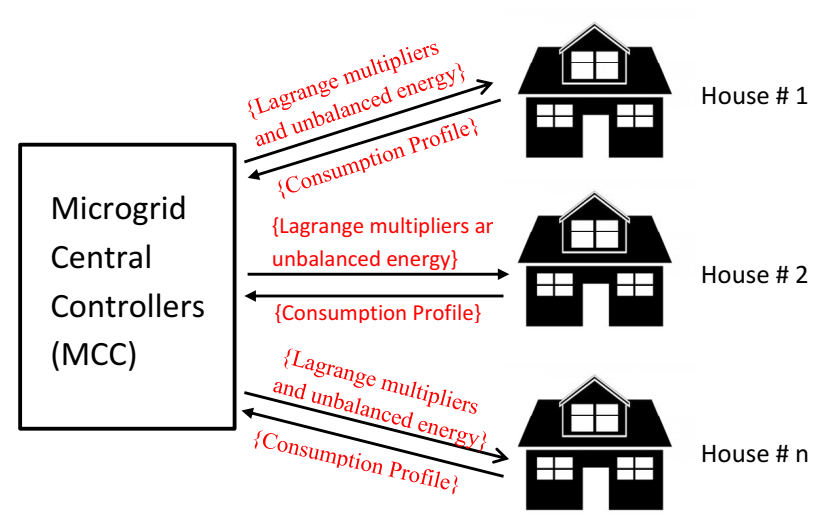

Figure 2: Exchange of Information between MCC and house BEMSs

Control and Communication (DECC) lab microgrid test system. The modified system includes various DERs, including a wind turbine, PV panel, fuel cell, microturbine, diesel generator and battery. The parameters for the dispatchable generators, PV, wind turbine and battery can be found in [18]. The three dispatchable generators are duplicated to make sure the microgrid can be operated in islanded mode without load shedding. The solar irradiance and temperature data is from [27]. The measured 1-minute data of Oak Ridge, Tennessee area on August, 1st, 2015 is used for the simulation. This is a typical summer day in southern states of the US. The cost of load shedding is set as $\$ 2.0 / \mathrm{kWh}$ and the amount of curtailed load should be less than $10 \%$ of the total non-HVAC load. The peak of non-HVAC load is about $180 \mathrm{~kW}$. Twenty houses are considered, each has a 5 $\mathrm{kW}$ HVAC system, which has a coefficient of performance (COP) $\eta_{h}=3$. The desired indoor temperature is set at $23{ }^{\circ} \mathrm{C}$ and the allowed deviation is $\pm 2{ }^{\circ} \mathrm{C}$. The customer discomfort factor is set at $\$ 0.05 /{ }^{\circ} \mathrm{C}$. All other parameters of the houses are taken from [25] (Table 7.1 in [25]). To represent the variety of the houses, the standard error of estimate in [25] (Table 7.1) is used to generate random errors for the parameters of each house. The modified DECC lab microgrid system includes various generations sources, such wind turbine, PV panel, fuel cell, microturbine and diesel generator as well as energy storage devices, such as battery. On the demand side, it considers multiple houses with different parameters. Thus, this modified DECC lab microgrid system represents the majority of both existing and future community microgrids.

The penalty parameter $\rho$ is set as 0.1 and the day-ahead market prices at PCC are used as the initial value of the Lagrangian multiplier in the distributed optimization. The analysis is conducted for a 24-hour scheduling horizon and the time interval is set to be 15 minutes. The load profile, market price and PV model are the same as in [18]. All 
numerical simulations are coded in MATLAB and solved using CPLEX 12.6. Six cases are studied as in Tab. I.

\section{A. Comparison of Cost Between Different Cases}

In order to show the benefit of integrating building thermal dynamics into community microgrid scheduling, we compare the total operating cost calculated in all cases. The total operating cost of the community microgrid consists of fuel cost, purchasing cost, battery degradation cost, voluntary load shedding cost and the cost associated with customer discomfort as in (3). In the case of autonomous control, the on/off state of HVAC system can be directly determined by solving equation (1) and performing the logic of temperature controlled relay. Then, the total cost can be calculated by scheduling the same microgrid with predetermined HVAC status. Comparing the total costs of centralized optimization in Case 3 and 4 with that of autonomous control in Case 1 and 2, the total costs are reduced by around $10 \%$ in both grid-connected and islanded modes by centralized optimization. This clearly indicates significant cost savings can be achieved by integrating building thermal dynamics and HVAC systems into the optimization. Comparing the total costs of distributed optimization in Case 5 and 6 with that of autonomous control in Case 1 and 2, the total costs are reduced by $6.7 \%$ in grid-connected mode and $4.5 \%$ in islanded mode by distributed optimization. The centralized optimization performs a little better than the distributed optimization. As mentioned earlier, due to the nonconvexity of subproblems, the global optimum cannot be guaranteed.

Table I: Total operating cost of the community microgrid in different cases

\begin{tabular}{|c|c|c|}
\hline \multicolumn{2}{|c|}{ Cases } & Cost (\$) \\
\hline \multirow{2}{*}{ Autonomous Control } & Case 1: Grid-connected & 984.08 \\
\cline { 2 - 3 } & Case 2: Islanded & 1106.67 \\
\hline \multirow{2}{*}{ Centralized Optimization } & Case 3: Grid-connected & 879.57 \\
\cline { 2 - 3 } & Case 4: Islanded & 994.28 \\
\hline \multirow{2}{*}{ Distributed Optimization } & Case 5: Grid-connected & 918.73 \\
\cline { 2 - 3 } & Case 6: Islanded & 1058.55 \\
\hline
\end{tabular}

\section{B. Operation in Grid-Connected Mode}

The results of community microgrid scheduling in gridconnected mode through different control and optimization methods are compared. Taking house \#1 for example, the indoor temperature and HVAC status are shown in Fig. 3. Comparing Fig. 3a with Fig. $3 b$ and 3c, it clearly shows how the centralized and distributed optimization model considering building thermal dynamics can precool the house to reduce the HVAC consumption during peak price intervals. Although the HVAC scheduling by centralized and distributed optimization are slightly different, the price signals of the distributed optimization converge to the dayahead price at PCC as shown in Fig. 3c. This is reasonable since the marginal unit of the community microgrid in gridconnected mode is the PCC.
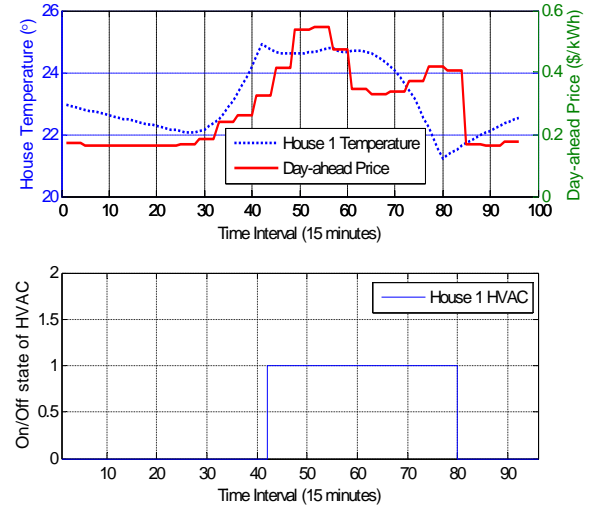

(a) Case 1: Autonomous control
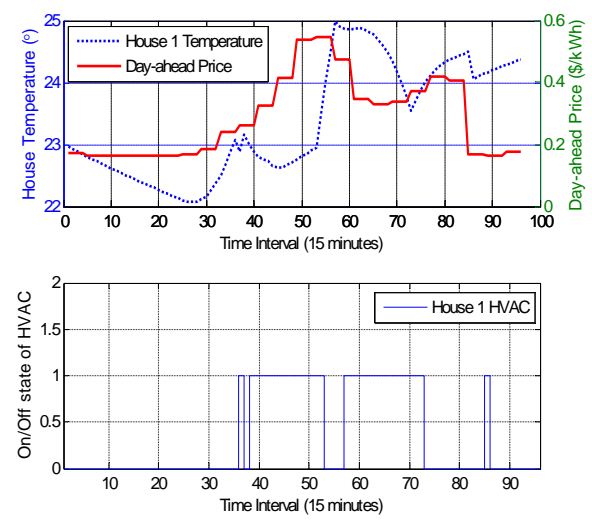

(b) Case 3: Centralized optimization
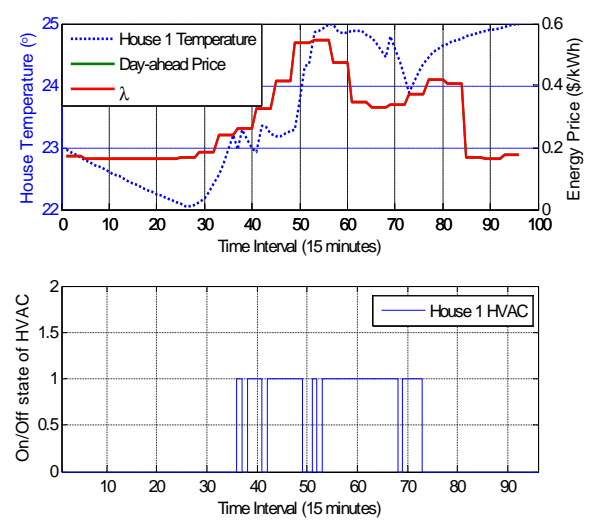

(c) Case 5: Distributed Optimization

Figure 3: House temperature and HVAC status in gridconnected mode

\section{Operation in Islanded Mode}

The results of community microgrid scheduling in islanded mode through different control and optimization methods are compared in Fig. 4. In islanded model, the microgrid net demand, i.e., the total load (both HVAC and 

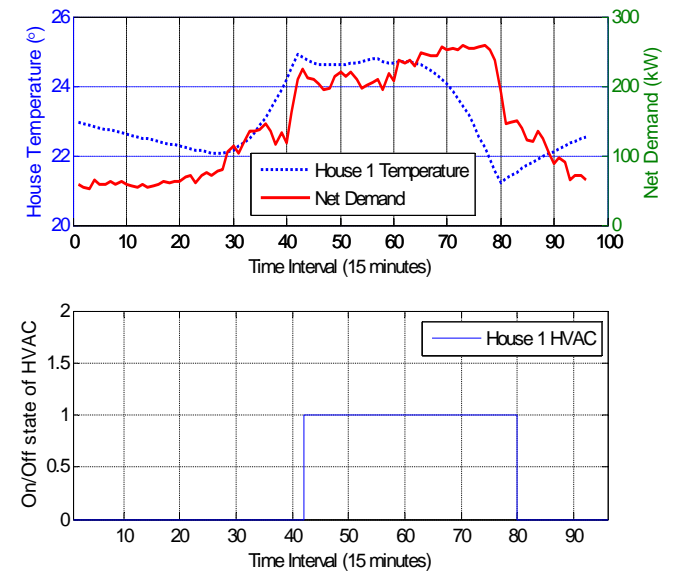

(a) Case 2: Autonomous control
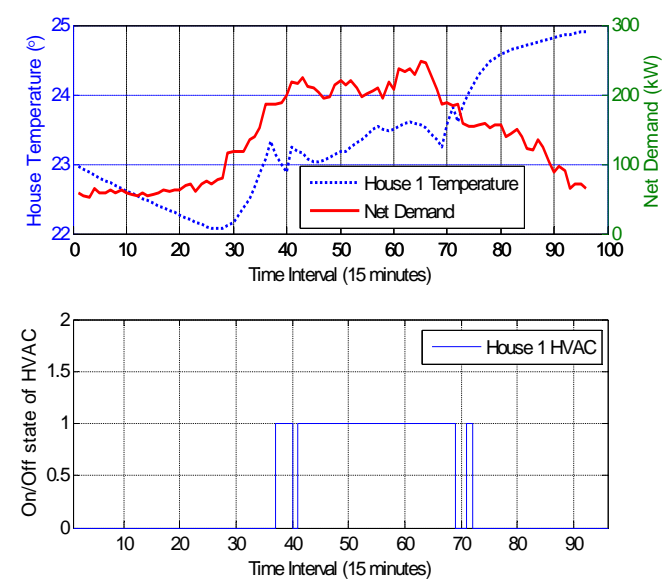

(b) Case 4: Centralized optimization
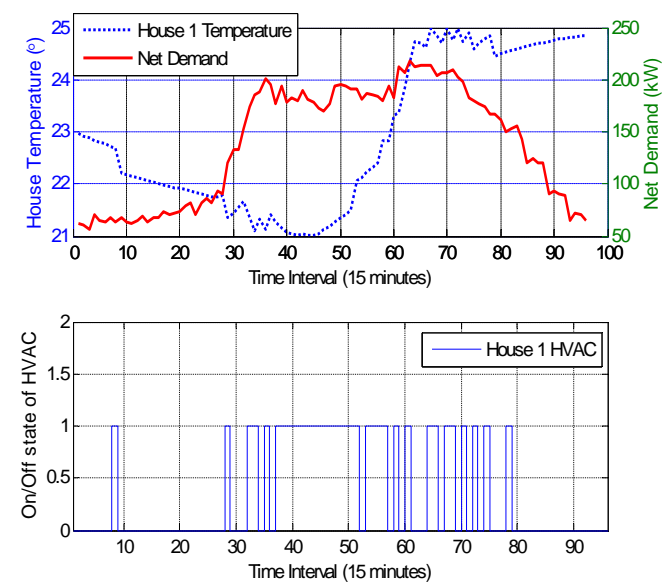

(c) Case 6: Distributed Optimization

Figure 4: House temperature and HVAC status in islanded mode
non-HVAC) minus the renewable generation is calculated The net demand is a good indicator of the energy price. With more net demand, units with higher marginal cost need to be committed. Thus, the energy price will be higher. As can be seen in Fig. 4, the peak demand of centralized and distributed optimization model considering building thermal dynamics has been reduced compared to autonomous control in Case 2. This indicates the peak energy prices of centralized and distributed optimization model are reduced compared to autonomous control. Comparing the centralized and distributed optimization model, we can see the peak demand of the distributed optimization model is further reduced, i.e. the peak energy price of distributed optimization is further reduced compared to centralized optimization.

Similar to the grid-connected cases, the centralized and distributed optimization model considering building thermal dynamics can precool the house to reduce the HVAC consumption during peak price/demand intervals. This effect is much more obvious in Case 6 as shown in 4c. In addition, the peak value of net demand is reduced in Case 6. It should also be noted that the HVAC scheduling by centralized and distributed optimization are different. This is because the distributed optimization converges to local optimum due to the nonconvexity of the subproblems.

\section{Convergence of Proposed Distributed Optimization}

For the distributed optimization Case 6, the Lagrangian multiplier and the microgrid net demand are shown as in Fig. 5. As can be seen, the Lagrangian multiplier and the microgrid net demand generally follow the same trend, except the two blue circles. The high prices during these intervals are due to the start-up cost of dispatchable generators. A small increase in the net demand can result in start-up of additional generators, which cause significant change of $\lambda$.

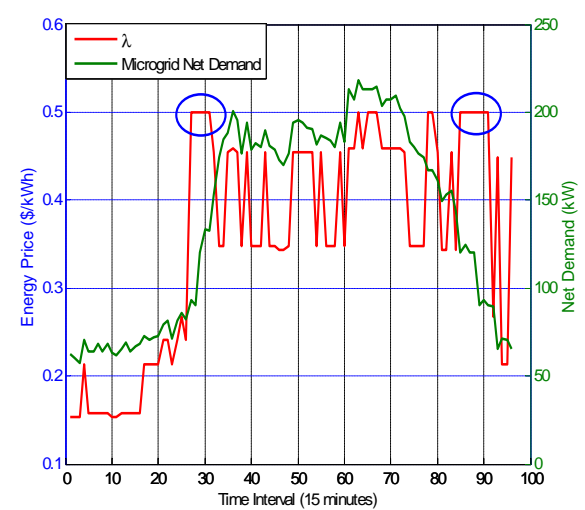

Figure 5: Lagrangian multiplier $\lambda$ and net demand of the microgrid in Case 6

For the proposed distributed optimization, a reasonable stopping criterion is that the primal residual has to be small, i.e., the total generation equals the total consumption [24]. 


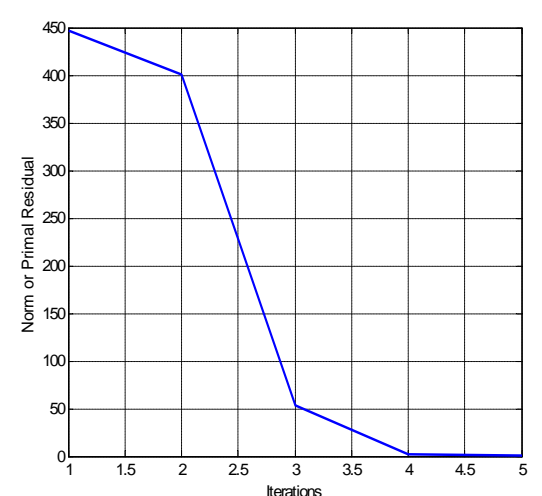

Figure 6: Norm of primal residual versus iteration

The norm of the primal residual as a function of the iteration number is shown in Fig. 6. The primal residual is calculated

$$
\begin{aligned}
& R^{\mathrm{P}}=\sum_{i=1}^{N_{G}} P_{i t}+P_{t}^{W}+P_{t}^{\mathrm{PV}}+P_{t}^{\mathrm{PCC}}+P_{t}^{\mathrm{LS}}-\sum_{j=1}^{N_{D}} P_{j t} \\
& +\sum_{b=1}^{N_{B}}\left(P_{b t}^{\mathrm{D}}-P_{b t}^{\mathrm{C}}\right)-\sum_{h=1}^{N_{H}} u_{h t} P_{h}^{H}
\end{aligned}
$$

\section{CONCLUSIONS}

In this paper, a new distributed optimization model for community microgrids considering building thermal dynamics is proposed. The total operating cost of community microgrids can be significantly reduced by integrating the building thermal dynamics and HVAC systems controller into BEMSs. The centralized optimization is decomposed to subproblems at MCC and each customer. The BEMSs of customers and the MCC exchange information on energy consumption and prices. ADMM is utilized to guarantee the convergence of the distributed optimization with nonconvex dual subproblems. Numerical simulations on the modified ORNL DECC microgrid show the effectiveness of the proposed distributed optimization model. The results of community microgrid scheduling through different control and optimization methods are compared.

\section{APPENDIX}

\section{NOMENCLATURE}

A symbol with $(\mathrm{k})$ on the upper right position stands for its value of $k$-th iteration. A bold symbol stands for its corresponding vector.

\section{A. Indices and Numbers}

$\begin{array}{ll}i & \text { Index of DGs, running from } 1 \text { to } N_{G} . \\ j & \text { Index of loads, running from } 1 \text { to } N_{D} . \\ b & \text { Index of batteries, running from } 1 \text { to } N_{B} . \\ h & \text { Index of houses, running from } 1 \text { to } N_{H} . \\ t & \text { Index of time periods, running from } 1 \text { to } N_{T} . \\ m & \text { Index of energy blocks offered by DGs, running } \\ & \text { from } 1 \text { to } N_{I} .\end{array}$

\section{B. Variables}

$u_{i t} \quad 1$ if DG $i$ is scheduled on during period $t$ and 0 otherwise.

$u_{h t} \quad 1$ if HVAC of house $h$ is scheduled on during period $t$ and 0 otherwise.

$u_{b t}^{\mathrm{C}}, u_{b t}^{\mathrm{D}} \quad 1$ if battery $b$ is scheduled charging/discharging during period $t$ and 0 otherwise.

$p_{i t}(m) \quad$ Power output scheduled from the $m$-th block of energy offer by DG $i$ during period $t$. Limited to $p_{i t}^{\max }(m)$.

$P_{i t} \quad$ Power output scheduled from DG $i$ during period $t$.

$P_{b t}^{\mathrm{C}}, P_{b t}^{\mathrm{D}} \quad$ Charging/discharging power of battery $b$ during period $t$.

$S O C_{b t} \quad$ State of charge of battery $b$ during period $t$.

$P_{t}^{\mathrm{PCC}} \quad$ Scheduled power at PCC during period $t$.

$P_{t}^{\mathrm{LS}} \quad$ Load curtailment during period $t$.

$T_{h t}^{\mathrm{In}} \quad$ Indoor temperature of house $h$ during period $t$.

$T_{h t}^{\mathrm{M}} \quad$ The temperature of thermal accumulating layer of inner walls and floor in house $h$ at period $t$.

$T_{h t}^{\mathrm{E}} \quad$ The temperature of house envelop at period $t$.

$\lambda_{t} \quad$ Lagrange multiplier of power balance equation during period $t$.

\section{Constants}

$\lambda_{i t}^{P}(m) \quad$ Marginal cost of the $m$-th block of energy offer $\lambda_{t} \quad$ by DG $i$ during period $t$.

$\lambda_{t}^{\mathrm{PCC}} \quad$ Purchasing price of energy from distribution grid during period $t$.

$\rho \quad$ Penalty parameter of augmented Lagrangian function.

$C_{b t} \quad$ Degradation cost of battery $b$ during period $t$.

$V_{t}^{\mathrm{LS}} \quad$ Cost of load curtailment during period $t$.

$P_{i}^{\max }, P_{i}^{\min }$ Maximum/minimum output of DG $i$.

$P_{t}^{\mathrm{W}} \quad$ Wind turbine power output during period $t$.

$P_{t}^{\mathrm{PV}} \quad$ PV power output during period $t$.

$P_{j t} \quad$ Power consumption scheduled for non-HVAC load $j$ during period $t$.

$P_{h}^{H} \quad$ Rated power of HVAC system in house $h$.

$P_{b}^{\mathrm{C} \text {, max }}, P_{b}^{\mathrm{D}, \text { max }}$ Maximum charging/discharging power of battery $b$.

$S O C_{b t}^{\max }, S O C_{b t}^{\min }$ Maximum/minimum state of charge of battery $b$ during period $t$.

$P_{t}^{\mathrm{LS}, \max } \quad$ Maximum load curtailment during period $t$.

$\eta_{b}^{\mathrm{C}}, \eta_{b}^{D} \quad$ Battery charging/discharging efficiency.

$\omega_{h t} \quad$ Customer discomfort cost of house $h$ during period $t$.

$\delta_{h t} \quad$ Allowed temperature deviation of house $h$ during period $t$.

$\kappa_{i} \quad$ Operating Cost of DG $i$ at the point of $P_{i}^{\min }$.

$T_{t}^{\mathrm{A}} \quad$ Ambient temperature during period $t$.

$T_{h t}^{\mathrm{D}} \quad$ Desired indoor temperature of house $h$ during period $t$. 
$\Phi_{t}$

$\eta_{h}$

Solar irradiance during period $t$.

Coefficient of performance (COP) of HVAC in house $h$.

\section{REFERENCES}

[1] CERTS Microgrid Concept, 2003, [Online]. Available: http://certs.lbl.gov/certs-der-micro.html.

[2] A. G. Madureira and J. A. Pecas Lopes, "Coordinated voltage support in distribution networks with distributed generation and microgrids," IET Renew. Power Gen., vol. 3, no. 4, pp. 439454, Dec. 2009.

[3] S. Beer, T. Gomez, D. Dallinger, I. Momber, C. Marnay, M. Stadler, and J. Lai, "An economic analysis of used electric vehicle batteries integrated into commercial building microgrids," IEEE Trans. Smart Grid, vol. 3, no. 1, pp. 517-525, Mar. 2012.

[4] A. G. Tsikalakis and N. D. Hatziargyriou, "Centralized control for optimizing microgrids operation," IEEE Trans. Energy Convers., vol. 23, no. 1, pp. 241-248, Mar. 2008.

[5] M. Agrawal and A. Mittal, "Micro grid technological activities across the globe: A review," Int. J. Res. Rev. Appl. Sci., vol. 7,no. 2, pp. 147-152, May 2011

[6] S. Gill, I. Kockar and G.W. Ault, "Dynamic Optimal Power Flow for Active Distribution Networks," IEEE Trans. Power Syst., vol. 29, no. 1, pp. 121-131, Jan. 201

[7] N. Daratha, B. Das and J. Sharma, "Coordination Between OLTC and SVC for Voltage Regulation in Unbalanced Distribution System Distributed Generation," IEEE Trans. Power Syst. , vol. 29, no. 1, pp. 289-299, Jan. 2014

[8] W. Gu, Z. Wu, R. Bo, W. Liu, G. Zhou, W. Chen, and A. $\mathrm{Wu}$, "Modeling, planning and optimal energy management of combined cooling, heating and power microgrid: A review," Int. J. Electr. Power Energy Syst., vol. 54, pp. 26-37, Jan. 2014.

[9] B. Belvedere, M. Bianchi, A. Borghetti, C. A. Nucci, M. Paolone, and A. Peretto, "A microcontroller-based power management system for standalone microgrids with hybrid power supply," IEEE Trans. Sustain. Energy, vol. 3, no. 3, pp. 422431, Jul. 2012

[10] H. Morais, P. Kádár, P. Faria, Z. A. Vale, and H. M. Khodr, "Optimal scheduling of a renewable micro-grid in an isolated load area using mixed-integer linear programming," Renew. Energy, vol. 35, no. 1, pp. 151-156, Jan. 2010.

[11] H. Babazadeh, W. Gao, Z. Wu, and Y. Li, "Optimal energy management of wind power generation system in islanded microgrid system," in Proc. North Amer. Power Symp. (NAPS), Sep. 2013, pp. 1-5.

[12] R. Palma-Behnke, C. Benavides, F. Lanas, B. Severino, L. Reyes, J. Llanos, and D. Sáez, "A microgrid energy management system based on the rolling horizon strategy," IEEE Trans. Smart Grid, vol. 4, no. 2, pp. 996-1006, Jun. 2013.

[13] A. Sobu and G. Wu, "Dynamic optimal schedule management method for microgrid system considering forecast errors of renewable power generations," in Proc. IEEE Int. Conf. Power Syst. Tech. (POWERCON), Nov. 2012, pp. 1-6.
[14] F. A. Mohamed and H. N. Koivo, "System modelling and online optimal management of MicroGrid using Mesh Adaptive Direct Search," Int. J. Electr. Power Energy Syst., vol. 32, no. 5, pp. 398-407, Jun. 2010.

[15] G. Cardoso, M. Stadler, A. Siddiqui, C. Marnay, N. DeForest, A. Barbosa-Póvoa, and P. Ferrão, "Microgrid reliability modeling and battery scheduling using stochastic linear programming," Electr. Power Syst. Res., vol. 103, pp. 61-69, Oct. 2013.

[16] W. Su, J. Wang, and J. Roh, "Stochastic Energy Scheduling in Microgrids With Intermittent Renewable Energy Resources," IEEE Trans. Smart Grid, vol. 5, no. 4, pp. 1876-1883, Jul. 2014.

[17] D. T. Nguyen and L. B. Le, "Optimal Bidding Strategy for Microgrids Considering Renewable Energy and Building Thermal Dynamics," IEEE Trans. Smart Grid, vol. 5, no. 4, pp. 1608-1620, Jul. 2014.

[18] G. Liu, Y. Xu, and K. Tomsovic, "Bidding Strategy for Microgrid in Day-Ahead Market Based on Hybrid Stochastic/Robust Optimization," IEEE Trans. Smart Grid, vol. 7, no. 1, pp. 227237, Jan. 2016.

[19] D. T. Nguyen and L. B. Le, "Joint optimization of electric vehicle and home energy scheduling considering user comfort preference," IEEE Trans. Smart Grid, vol. 5, no. 1, pp. 188-199, Jan. 2014.

[20] D. T. Nguyen and L. B. Le, "Optimal Bidding Strategy for Microgrids Considering Renewable Energy and Building Thermal Dynamics," IEEE Trans. Smart Grid, vol. 5, no. 4, pp. 1608-1620, July 2014.

[21] N. Gatsis and G. B. Giannakis, "Residential load control: Distributed scheduling and convergence with lost AMI messages," IEEE Trans. Smart Grid, vol. 3, no. 2, pp. 770-786, Jun. 2012.

[22] B. Moradzadeh and K. Tomsovic, "Two-Stage Residential Energy Management Considering Network Operational Constraints," IEEE Transactions on Smart Grid, vol. 4, no. 4, pp. 2339-2346, Dec. 2013.

[23] A. Khodaei and M. Shahidehpour, "Optimal operation of a community- based microgrid," in Proc. IEEE PES ISGT Asia, 2011, pp. 1-3.

[24] S. Boyd, N. Parikh, E. Chu, B. Peleato, E. Eckstein, "Distributed Optimization and Statistical Learning via the Alternating Direction Method of Multipliers" Foundations and Trends® in Machine Learning, vol. 3, pp. 1-122, Jan. 2011.

[25] A. Thavlov, "Dynamic optimization of power consumption," M.S. thesis, Tech. Univ. Denmark, Kongens Lyngby, Denmark, 2008.

[26] M. Carrión, and J. M. Arroyo, "A computationally efficient mixed-integer linear formulation for the thermal unit commitment problem," IEEE Trans. Power Syst., vol. 21, no. 3, pp. 1371-1378, Aug. 2006.

[27] [Online]. Available: http://www.nrel.gov/midc/ornl_rsr/ 